\title{
Existence of periodic solutions of a Liénard equation with a singularity of repulsive type
}

\author{
Shiping Lu, Yajiao Wang* and Yuanzhi Guo
}

\section{"Correspondence:} 945714244@qq.com

College of Math \& Statistics, Nanjing University of Information Science \&

Technology, Nanjing, 210044, China

\begin{abstract}
In this paper, the problem of positive periodic solutions is studied for the Liénard equation with a singularity of repulsive type,

$$
x^{\prime \prime}+f(x) x^{\prime}-\frac{\alpha(t)}{x^{\mu}}=h(t)
$$

where $f:(0,+\infty) \rightarrow R$ is continuous, $\alpha, h$ are continuous with $T$-periodic and $\alpha(t) \geq 0$ for all $t \in R, \mu \in(0,+\infty)$ is a constant. By means of a Manásevich-Mawhin's continuation theorem, a sufficient and necessary condition is obtained for the existence of positive $T$-periodic solutions of the equation. The interesting point is that the weak singularity of restoring force $\frac{\alpha(t)}{x^{\mu}}$ at $x=0$ is allowed and $f$ may have a singularity at $x=0$.
\end{abstract}

Keywords: Liénard equation; Manásevich-Mawhin's continuation theorem; singularity; periodic solution

\section{Introduction}

In the past years, much attention from researchers in differential equations was paid to investigating the problem of periodic solutions for second order differential equations with singularities. This is due to the fact that the singularity has a significant background in applied sciences and physics (see [1-12] and the references therein). The first study on the periodic problem for second order singular differential equations seems to be the work of Nagumo [13] in 1943. After some work [14-16], the interest increased in this area with the pioneering paper of Lazer and Solimini [17]. They considered the existence of periodic solutions for the equation with a singularity of repulsive type,

$$
x^{\prime \prime}-\frac{1}{x^{\alpha}(t)}=h(t),
$$

where $h: R \rightarrow R$ is continuous with $T$-periodic, $\alpha \in(0,+\infty)$ is a constant. For $\alpha \in[1,+\infty)$ (called the strong force condition), by using topological degree methods, they found that the necessary and sufficient condition for the existence of positive periodic solutions for equation (1.1) is

$$
\bar{h}:=\frac{1}{T} \int_{0}^{T} h(s) d s<0 .
$$

(c) The Author(s) 2017. This article is distributed under the terms of the Creative Commons Attribution 4.0 International License (http://creativecommons.org/licenses/by/4.0/), which permits unrestricted use, distribution, and reproduction in any medium, provided you give appropriate credit to the original author(s) and the source, provide a link to the Creative Commons license, and indicate if changes were made. 
For $\alpha \in(0,1)$ (weak singularity condition), they produced some examples of $h(t)$ with $\bar{h}<0$ and such that equation (1.1) does not have any positive $T$-periodic solution. After that, the strong force condition $\alpha \geq 1$ was regarded as crucial assumption in [18-24]. By using some fixed point theorems in cones, the existence of periodic solutions has been widely studied recently for the conservative equation of repulsive type,

$$
x^{\prime \prime}+a(t) x-\frac{b(t)}{x^{\alpha}(t)}=c(t)
$$

where $a, b, c \in L^{1}[0, T]$ with $a(t) \geq 0, b(t) \geq 0$ for a.e. $t \in[0, T]$ and being positive in a set of positive measure [25-29]. Most of them focus on the case in which the singular nonlinearity was allowed to have a weak singularity $(\alpha \in(0,1))$. Compared with the study of conservative equations with weak singularities, the corresponding ones of the Liénard equation with a weak singularity of repulsive type is considerably neglected. We find that the strong singularity is needed in the most recent papers associated to singular Liénard equation of repulsive type [30-35]. For example, Jebelean and Mawhin in [11] considered the problem of the existence of positive periodic solutions for a $p$-Laplacian Liénard equation like

$$
\left(\left|x^{\prime}\right|^{p-2} x^{\prime}\right)^{\prime}+f(x) x^{\prime}-\frac{\beta}{x^{\mu}}=h(t),
$$

where $p>1, \beta>0, \mu>0$ are constants, $f:[0,+\infty) \rightarrow \mathbb{R}$ is continuous, $h: \mathbb{R} \rightarrow \mathbb{R}$ is a $T$-periodic function with $h \in L^{\infty}[0, T]$. Under the condition of strong singularity $\mu \geq 1$, they found that the necessary and sufficient condition for the existence of positive periodic solutions for equation (1.3) is $\bar{h}<0$. Wang in [32] further studied the existence of positive periodic solutions for a delay Liénard equation with a strong singularity $(\mu \in[1,+\infty))$ of repulsive type,

$$
x^{\prime \prime}+f(x) x^{\prime}+a(t) x(t-\tau)-\frac{\beta}{x^{\mu}(t-\tau)}=h(t)
$$

Hakl, Torres and Zamora in [33] considered the periodic problem for the singular equation of repulsive type,

$$
u^{\prime \prime}(t)+f(u(t)) u^{\prime}(t)+\varphi(t)(u(t))^{\delta}+g(u(t))=0,
$$

where $\delta \in(0,1]$ is a constant, $\varphi$ is a $T$-periodic function with $\varphi \in L([0, T], \mathbb{R}), f \in$ $C((0,+\infty), \mathbb{R})$ may be singular at $x=0, g \in C((0,+\infty), \mathbb{R})$ has a repulsive singularity at $x=0$, i.e., $g(x) \rightarrow-\infty$ as $x \rightarrow 0^{+}$. By using Schauder's fixed point theorem, some results on the existence of positive $T$-periodic solutions are obtained. However, a strong singularity, $\int_{0}^{1} g(s) d s=-\infty$, is also required.

Now, the question is how to study the periodic problem of equation (1.5) under the condition of weak singularity $\int_{0}^{1} g(s) d s>-\infty$. Motivated by this, the purpose of this paper is to investigate the existence of positive $T$-periodic solutions for Liénard equation with a singularity of repulsive type

$$
x^{\prime \prime}+f(x) x^{\prime}-\frac{\alpha(t)}{x^{\mu}}=h(t)
$$


where $f:(0,+\infty) \rightarrow R$ is continuous, $\alpha, h$ are continuous $T$-periodic functions with $\alpha(t) \geq 0$ for all $t \in R$ and $\alpha(t) \not \equiv 0, \mu \in(0,+\infty)$ is a constant. By using a continuation theorem established by Mawhin and Manásevich [36], some new results are obtained. The interesting point is that the weak singularity of restoring force term $\frac{\alpha(t)}{x^{\mu}}$ at $x=0$ is allowed and $f$ may have a singularity at $x=0$. Furthermore, under the condition of $\int_{0}^{1} f(s) d s=\infty$, a sufficient and necessary condition is obtained for the existence of positive $T$-periodic solutions of equation (1.6).

\section{Preliminary lemmas}

Let $C_{T}=x \in C(\mathbb{R}, \mathbb{R}): x(t+T)=x(t)$ for all $t \in \mathbb{R}$ with the norm defined by $|x|_{\infty}=$ $\max _{t \in[0, T]}|x(t)|$. For any $T$-periodic solution $h(t)$ with $h \in C_{T}, h_{+}(t)$ and $h_{-}(t)$ is denoted by $\max \{(h(t), 0)\}$ and $-\min \{(h(t), 0)\}$, respectively, and $\bar{h}=\frac{1}{T} \int_{0}^{T} h(s) d s$. Clearly, $h(t)=h_{+}(t)-h_{-}(t)$ for all $t \in \mathbb{R}$, and $\bar{h}=\bar{h}_{+}-\bar{h}_{-}$. Furthermore, $\|\varphi\|_{p}:=\left(\int_{0}^{T}|\varphi(t)|^{p} d t\right)^{\frac{1}{p}}$, $p \in[1,+\infty), \varphi \in C_{T}$.

The following lemma is a corollary of Theorem 3.1 in [36].

Lemma 2.1 Assume that there exist positive constants $M_{0}, M_{1}$ and $M_{2}$ with $0<M_{0}<M_{1}$, such that the following conditions hold:

1. for each $\lambda \in(0,1]$, each possible positive $T$-periodic solution $x$ to the equation

$$
u^{\prime \prime}+\lambda f(u) u^{\prime}-\lambda \frac{\alpha(t)}{u^{\mu}}=\lambda h(t)
$$

satisfies the inequalities $M_{0}<x(t)<M_{1}$ and $\left|x^{\prime}(t)\right|<M_{2}$ for all $t \in[0, T]$;

2. each possible solution $x \in(0,+\infty)$ to the equation

$$
\frac{\bar{\alpha}}{x^{\mu}}+\bar{h}=0
$$

satisfies the inequality $M_{0}<x<M_{1}$;

3. the inequality

$$
\left(\frac{\bar{\alpha}}{M_{0}^{\mu}}+\bar{h}\right)\left(\frac{\bar{\alpha}}{M_{1}^{\mu}}+\bar{h}\right)<0
$$

holds. Then equation (1.6) has at least one positive T-periodic solution $x(t)$ such that $M_{0}<$ $x(t)<M_{1}$ for all $t \in[0, T]$.

Lemma 2.2 ([29]) Let $x(t)$ be a continuously differentiable T-periodic function. Then, for any $\tau \in[0, T]$,

$$
\left(\int_{0}^{T} x^{2}(t) d t\right)^{\frac{1}{2}} \leq \frac{T}{\pi}\left(\int_{0}^{T} x^{\prime 2}(t) d t\right)^{\frac{1}{2}}+\sqrt{T}|x(\tau)| .
$$

In order to study the existence of positive periodic solutions to equation (1.6), we list the following assumptions:

$\left(\mathrm{H}_{1}\right) \lim _{x \rightarrow 0^{+}}\left|\int_{x}^{1} f(s) d s\right|=+\infty$.

$\left(\mathrm{H}_{2}\right)\left(-\frac{\bar{\alpha}}{\bar{h}}\right)^{\frac{1}{\mu}}>T^{\frac{1}{2}}\left[\frac{T}{\pi}\|h\|_{2}+\left(T^{\frac{1}{2}}\left(-\frac{\bar{\alpha}}{\bar{h}}\right)^{\frac{1}{\mu}}\|h\|_{2}\right)^{\frac{1}{2}}\right]$. 
Remark 2.1 If $\bar{h}<0$, then there are constants $D_{1}$ and $D_{2}$ with $0<D_{1}<D_{2}$ such that

$$
\frac{\bar{\alpha}}{x^{\mu}}+\bar{h}>0 \quad \forall x \in\left(0, D_{1}\right)
$$

and

$$
\frac{\bar{\alpha}}{x^{\mu}}+\bar{h}<0 \quad \forall x \in\left(D_{2}, \infty\right)
$$

Now, we embed equation (1.6) into the following equations family with a parameter $\lambda \in(0,1]$,

$$
x^{\prime \prime}+\lambda f(x) x^{\prime}-\lambda \frac{\alpha(t)}{x^{\mu}}=\lambda h(t), \quad \lambda \in(0,1] .
$$

\section{Main results}

Theorem 3.1 If $\left(\mathrm{H}_{1}\right)$ holds, then equation (1.6) has a positive T-periodic solution if and only if $\bar{h}<0$.

Proof Suppose that equation (1.6) has a positive $T$-periodic solution $y(t)$, then

$$
y^{\prime \prime}+f(y) y^{\prime}-\frac{\alpha(t)}{y^{\mu}}=h(t)
$$

Integrating (3.1) on the interval $[0, T]$, and by using

$$
\int_{0}^{T} y^{\prime \prime}(s) d s=\int_{0}^{T} f(y(s)) y^{\prime}(s) d s=0
$$

we have

$$
\int_{0}^{T} \frac{\alpha(s)}{y^{\mu}(s)} d s=-T \bar{h}
$$

which together with the assumption of $\alpha(t) \geq 0$ and $y(t)>0$ for all $t \in[0, T]$ gives a necessary condition for the existence of a positive $T$-periodic solution of equation (1.6): $\bar{h}<0$.

Below, we will show the proof of sufficiency. In order to do it, suppose that $\bar{h}<0$, and let $u$ be an arbitrary positive $T$-periodic solution of (2.1). Then

$$
u^{\prime \prime}+\lambda f(u) u^{\prime}-\lambda \frac{\alpha(t)}{u^{\mu}}=\lambda h(t), \quad \lambda \in(0,1]
$$

Integrating (3.2) over the interval $[0, T]$, we have

$$
\int_{0}^{T} \frac{\alpha(t)}{u^{\mu}} d t=-\int_{0}^{T} h(t) d t=-T \bar{h}
$$

Due to the fact that $\alpha(t)$ is non-negative, $\frac{1}{u_{M}^{\mu}} \int_{0}^{T} \alpha(t) d t \leq \int_{0}^{T} \frac{\alpha(t)}{u^{\mu}(t)} d t \leq \frac{1}{u_{m}^{\mu}} \int_{0}^{T} \alpha(t) d t$, where $u_{m}, u_{M}$ are the global minimum and maximum, respectively, of $u$. Then there is a point $\eta \in[0, T]$ such that

$$
\frac{1}{u^{\mu}(\eta)} \int_{0}^{T} \alpha(t) d t=-T \bar{h}
$$


which results in

$$
T \bar{\alpha}=-u^{\mu}(\eta) T \bar{h},
$$

and then

$$
u(\eta)=\left(-\frac{\bar{\alpha}}{\bar{h}}\right)^{\frac{1}{\mu}}
$$

Multiplying (3.2) with $u(t)$, and integrating it over the interval $[0, T]$, we obtain

$$
\int_{0}^{T} u^{\prime \prime}(t) u(t) d t-\lambda \int_{0}^{T} \frac{\alpha(t) u(t)}{u^{\mu}(t)} d t=\lambda \int_{0}^{T} h(t) u(t) d t
$$

By using $\int_{0}^{T} u^{\prime \prime}(t) u(t) d t=-\int_{0}^{T}\left|u^{\prime}(t)\right|^{2} d t$, we have

$$
\int_{0}^{T}\left|u^{\prime}(t)\right|^{2} d t+\lambda \int_{0}^{T} \alpha(t) u^{1-\mu}(t) d t=-\lambda \int_{0}^{T} h(t) u(t) d t
$$

which together with the fact of $\alpha(t) \geq 0$ for all $t \in[0, T]$ and $\alpha(t) \not \equiv 0$ gives

$$
\int_{0}^{T}\left|u^{\prime}(t)\right|^{2} d t<\int_{0}^{T}|h(t)| u(t) d t \leq\left(\int_{0}^{T} h^{2}(t) d\right)^{\frac{1}{2}}\left(\int_{0}^{T} u^{2}(t) d t\right)^{\frac{1}{2}}
$$

By Lemma 2.2, we have

$$
\left(\int_{0}^{T} u^{2}(t) d t\right)^{\frac{1}{2}}<\frac{T}{\pi}\left(\int_{0}^{T}\left|u^{\prime}(t)\right|^{2} d t\right)^{\frac{1}{2}}+\sqrt{T} u(\eta)
$$

It follows from (3.4) that

$$
\left(\int_{0}^{T} u^{2}(t) d t\right)^{\frac{1}{2}}<\frac{T}{\pi}\left(\int_{0}^{T}\left|u^{\prime}(t)\right|^{2} d t\right)^{\frac{1}{2}}+\sqrt{T}\left(-\frac{\bar{\alpha}}{\bar{h}}\right)^{\frac{1}{\mu}} .
$$

Substituting it into (3.5), we have

$$
\begin{aligned}
\int_{0}^{T}\left|u^{\prime}(t)\right|^{2} d t & <\left(\int_{0}^{T} h^{2}(t) d t\right)^{\frac{1}{2}}\left[\frac{T}{\pi}\left(\int_{0}^{T}\left|u^{\prime}(t)\right|^{2} d t\right)^{\frac{1}{2}}+\sqrt{T}\left(-\frac{\bar{\alpha}}{\bar{h}}\right)^{\frac{1}{\mu}}\right] \\
& =\frac{T}{\pi}\left(\int_{0}^{T} h^{2}(t) d t\right)^{\frac{1}{2}}\left(\int_{0}^{T}\left|u^{\prime}(t)\right|^{2} d t\right)^{\frac{1}{2}}+T^{\frac{1}{2}}\left(-\frac{\bar{\alpha}}{\bar{h}}\right)^{\frac{1}{\mu}}\left(\int_{0}^{T} h^{2}(t) d t\right)^{\frac{1}{2}},
\end{aligned}
$$

by the inequality $X^{2}-A X-B<0$, we can obtain $X<A+B^{\frac{1}{2}}$. Let $X=\left(\int_{0}^{T}\left|u^{\prime}(t)\right|^{2} d t\right)^{\frac{1}{2}}$, $A=\frac{T}{\pi}\left(\int_{0}^{T} h^{2}(t) d t\right)^{\frac{1}{2}}, B=T^{\frac{1}{2}}\left(-\frac{\bar{\alpha}}{h}\right)^{\frac{1}{\mu}}\left(\int_{0}^{T} h^{2}(t) d t\right)^{\frac{1}{2}}$, we have

$$
\left(\int_{0}^{T}\left|u^{\prime}(t)\right|^{2} d t\right)^{\frac{1}{2}}<\frac{T}{\pi}\|h\|_{2}+T^{\frac{1}{4}}\left(-\frac{\bar{\alpha}}{\bar{h}}\right)^{\frac{1}{2 \mu}}\|h\|_{2}^{\frac{1}{2}}:=\rho_{1} .
$$


Combined (3.4) with (3.6), we have

$$
u(t) \leq u(\eta)+\sqrt{T}\left\|u^{\prime}\right\|_{2}<\left(-\frac{\bar{\alpha}}{\bar{h}}\right)^{\frac{1}{\mu}}+T^{\frac{1}{2}} \rho_{1}:=M .
$$

Let $t_{1}, t_{2}$ be the maximum point and the minimum point of $u(t)$ on $[0, T]$, respectively, then

$$
\int_{t_{1}}^{t_{2}} u^{\prime \prime}(t) d t+\lambda \int_{t_{1}}^{t_{2}} f(u(t)) u^{\prime}(t) d t-\lambda \int_{t_{1}}^{t_{2}} \frac{\alpha(t)}{u^{\mu}(t)} d t=\lambda \int_{t_{1}}^{t_{2}} h(t) d t
$$

which together with $u^{\prime}\left(t_{1}\right)=u^{\prime}\left(t_{2}\right)=0$ yields

$$
F\left(u\left(t_{2}\right)\right)-F\left(u\left(t_{1}\right)\right)=\int_{t_{1}}^{t_{2}} \frac{\alpha(t)}{u^{\mu}(t)} d t+\int_{t_{1}}^{t_{2}} h(t) d t
$$

where $F(x)=\int_{1}^{x} f(s) d s$, and then

$$
\left|F\left(u\left(t_{2}\right)\right)\right| \leq\left|F\left(u\left(t_{1}\right)\right)\right|+\int_{0}^{T} \frac{\alpha(t)}{u^{\mu}(t)} d t+\int_{0}^{T}|h(t)| d t .
$$

It follows from (3.4) and (3.7) that

$$
\begin{aligned}
\left|F\left(u\left(t_{2}\right)\right)\right| & \leq \max _{\left(-\frac{\bar{\alpha}}{\bar{h}}\right)^{\frac{1}{\mu}} \leq z \leq M}|F(z)|-T \bar{h}+T \overline{h \mid} \\
& \leq \max _{\left(-\frac{\bar{\alpha}}{\bar{h}}\right)^{\frac{1}{\mu}} \leq z \leq M}|F(z)|+2 T \bar{h}_{-} .
\end{aligned}
$$

It is easy to see from $\left(\mathrm{H}_{1}\right)$ that there is a constant $\gamma_{0}>0$, such that

$$
|F(z)|=\left|\int_{1}^{z} f(s) d s\right|>\max _{\left(-\frac{\bar{\alpha}}{\bar{h}}\right)^{\frac{1}{\mu}} \leq z \leq M}|F(z)|+2 T \bar{h}_{-} \quad \text { for } z \in\left(0, \gamma_{0}\right] .
$$

By (3.8), we have

$$
u\left(t_{2}\right)>\gamma_{0},
$$

and then

$$
u(t)>\gamma_{0} \quad \text { for all } t \in[0, T]
$$

Next, if $u$ attains its maximum over $[0, T]$ at $t_{1} \in[0, T]$, then $u^{\prime}\left(t_{1}\right)=0$ and we see from (3.2) that

$$
u^{\prime}(t)=\lambda \int_{t_{1}}^{t}\left[-f(u) u^{\prime}+\frac{\alpha(t)}{u^{\mu}}+h(t)\right] d t \quad \text { for all } t \in\left[t_{1}, t_{1}+T\right]
$$


Therefore,

$$
\begin{aligned}
\left|u^{\prime}(t)\right| & \leq \lambda\left|F(u(t))-F\left(u\left(t_{1}\right)\right)\right|+\lambda \int_{t_{1}}^{t_{1}+T} \frac{\alpha(t)}{u^{\mu}(t)} d t+\lambda \int_{t_{1}}^{t_{1}+T} h_{+}(s) d s \\
& \leq 2 \lambda \max _{\gamma_{0} \leq u \leq M}|F(u)|+\lambda \int_{0}^{T} \frac{\alpha(t)}{u^{\mu}(t)} d t+T \bar{h}_{+} .
\end{aligned}
$$

Substituting (3.3) into (3.10), we have

$$
\left|u^{\prime}(t)\right| \leq 2 \max _{\gamma_{0} \leq u \leq M}|F(u)|-T \bar{h}+T \bar{h}_{+}:=M^{*} \quad \text { for all } t \in[0, T]
$$

and then

$$
\left|u^{\prime}(t)\right| \leq M^{*} \quad \text { for all } t \in[0, T]
$$

From (3.7), (3.9), (3.11) and Remark 2.1, we can choose $M_{0}:=\min \left\{\gamma_{0}, D_{1}\right\}$ where $D_{1}$ is determined in Remark 2.1, $M_{1}=M$ and $M_{2}=M^{*}$ such that all the conditions of Lemma 2.1 are satisfied. Thus, by using Lemma 2.1, we see that equation (1.6) has at least one positive $T$-periodic solution. The proof is complete.

Theorem 3.2 Suppose that $\bar{h}<0$ and $\left(\mathrm{H}_{2}\right)$ holds. Then equation (1.6) has at least one positive T-periodic solution.

Proof Suppose that $u$ is an arbitrary positive $T$-periodic solution of equation (2.1), then

$$
u^{\prime \prime}+\lambda f(u) u^{\prime}-\lambda \frac{\alpha(t)}{u^{\mu}}=\lambda h(t), \quad \lambda \in(0,1]
$$

Similar to the proof of (3.4) and (3.6), we find that there is a point $\xi \in[0, T]$ such that

$$
u(\xi)=\left(-\frac{\bar{\alpha}}{\bar{h}}\right)^{\frac{1}{\mu}}
$$

and

$$
\left(\int_{0}^{T}\left|u^{\prime}(t)\right|^{2} d t\right)^{\frac{1}{2}}<\frac{T}{\pi}\|h\|_{2}+T^{\frac{1}{4}}\left(-\frac{\bar{\alpha}}{\bar{h}}\right)^{\frac{1}{2 \mu}}\|h\|_{2}^{\frac{1}{2}}:=\rho_{1} .
$$

In view of the inequality

$$
\begin{aligned}
u(t) & =u(\xi)+\int_{\xi}^{t} u^{\prime}(s) d s \leq u(\xi)+\int_{\xi}^{\xi+T}\left|u^{\prime}(s)\right| d s \\
& =u(\xi)+\int_{0}^{T}\left|u^{\prime}(s)\right| d s, \quad t \in[\xi, \xi+T],
\end{aligned}
$$

and by using (3.13), together with Schwarz inequality, we have

$$
\max _{t \in[0, T]} u(t) \leq\left(-\frac{\bar{\alpha}}{\bar{h}}\right)^{\frac{1}{\mu}}+T^{\frac{1}{2}}\left(\int_{0}^{T}\left|u^{\prime}(s)\right|^{2} d s\right)^{\frac{1}{2}}
$$


and

$$
\min _{t \in[0, T]} u(t) \geq u(\xi)-\left|\int_{\xi}^{t} u^{\prime}(s) d s\right| \geq\left(-\frac{\bar{\alpha}}{\bar{h}}\right)^{\frac{1}{\mu}}-T^{\frac{1}{2}}\left(\int_{0}^{T}\left|u^{\prime}(s)\right|^{2} d s\right)^{\frac{1}{2}}
$$

Substituting (3.14) into (3.15) and (3.16), respectively, we have

$$
\max _{t \in[0, T]} u(t) \leq\left(-\frac{\bar{\alpha}}{\bar{h}}\right)^{\frac{1}{\mu}}+T^{\frac{1}{2}}\left[\frac{T}{\pi}\|h\|_{2}+T^{\frac{1}{4}}\left(-\frac{\bar{\alpha}}{\bar{h}}\right)^{\frac{1}{2 \mu}}\|h\|_{2}^{\frac{1}{2}}\right]
$$

and

$$
\min _{t \in[0, T]} u(t) \geq\left(-\frac{\bar{\alpha}}{\bar{h}}\right)^{\frac{1}{\mu}}-T^{\frac{1}{2}}\left[\frac{T}{\pi}\|h\|_{2}+T^{\frac{1}{4}}\left(-\frac{\bar{\alpha}}{\bar{h}}\right)^{\frac{1}{2 \mu}}\|h\|_{2}^{\frac{1}{2}}\right] .
$$

The rest of the proof works almost analogously to the corresponding ones of Theorem 3.1.

Example 3.1 Considering the following equation:

$$
x^{\prime \prime}(t)+\frac{x^{\prime}(t)}{x^{2}(t)}-\frac{\sin ^{2} t}{x^{\frac{2}{3}}}=-1+\cos t .
$$

Corresponding to equation (1.6), we have $f(x)=\frac{1}{x^{2}}, \mu=\frac{2}{3}, \alpha(t)=\sin ^{2} t, h(t)=-1+\cos t$, and then $T=2 \pi, \int_{0}^{1} f(s) d s=+\infty$. This implies that assumption $\left(\mathrm{H}_{1}\right)$ holds. Since $\bar{h}=-1<0$, by using Theorem 3.1, we find that equation (3.19) has at least one positive $2 \pi$-periodic solution.

Example 3.2 Considering the following equation:

$$
x^{\prime \prime}(t)+\frac{x^{\prime}(t)}{x^{\frac{1}{2}}(t)}-\frac{\sin ^{2} 8 t}{x^{\frac{3}{4}}}=-\cos ^{2} 8 t .
$$

Corresponding to equation (1.6), $f$ can be regarded as $f(x)=\frac{1}{x^{\frac{1}{2}}}, \mu=\frac{3}{4}, \alpha(t)=\sin ^{2} 8 t$ and $h(t)=-\cos ^{2} 8 t$. Since $\int_{0}^{1} f(s) d s=2$, it follows that assumption $\left(\mathrm{H}_{1}\right)$ does not hold. This implies that Theorem 3.1 cannot be used to study the existence of periodic solutions to (3.20). But, by simple calculating, we can verify that

$$
-\frac{\bar{h}}{\bar{\alpha}}=1, \quad\|h\|_{2}^{2}=\frac{3 T}{8},
$$

where $T=\frac{\pi}{8}$, and then

$$
\left(-\frac{\bar{\alpha}}{\bar{h}}\right)^{\frac{1}{\mu}}-T^{\frac{1}{2}}\left[\frac{T}{\pi}\|h\|_{2}+\left(T^{\frac{1}{2}}\left(-\frac{\bar{\alpha}}{\bar{h}}\right)^{\frac{1}{\mu}}\|h\|_{2}\right)^{\frac{1}{2}}\right]=1-\left(\frac{\sqrt{6} \pi}{256}+\frac{\sqrt[4]{6} \pi}{16}\right)>0
$$

which implies that assumption $\left(\mathrm{H}_{2}\right)$ holds. Thus, by using Theorem 3.2, we find that (3.20) has at least one positive $\frac{\pi}{8}$-periodic solution. 
Remark The above two examples can neither be studied by using the results in [31, 32, 34] and [35], since $f(x)$ in (3.19) and in (3.20) are all singular at $x=0$, nor be studied by using the results in [33], since the restoring force terms of $\frac{\sin ^{2} t}{x^{\frac{2}{3}}}$ in (3.19) and $\frac{\sin ^{2} 8 t}{x^{\frac{3}{4}}}$ in (3.20) have weak singularities at $x=0$.

\section{Acknowledgements}

The authors thank the referees for valuable comments. This research is supported by the NSF of China (No. 11271197).

\section{Competing interests}

The authors declare that they have no competing interests.

Authors' contributions

All the authors read and approved the final manuscript.

\section{Publisher's Note}

Springer Nature remains neutral with regard to jurisdictional claims in published maps and institutional affiliations.

Received: 5 April 2017 Accepted: 13 June 2017 Published online: 27 June 2017

\section{References}

1. Lei, J, Zhang, M: Twist property of periodic motion of an atom near a charged wire. Lett. Math. Phys. 60(1), 9-17 (2002)

2. Bevc, V, Palmer, JL, Süsskind, C: On the design of the transition region of axi-symmetric magnetically focused beam valves. J. Br. Inst. Radio Eng. 18, 696-708 (1958)

3. Ye, Y, Wang, X: Nonlinear differential equations in electron beam focusing theory. Acta Math. Appl. Sin. 1, 13-41 (1978) (in Chinese)

4. Huang, J, Ruan, S, Song, J: Bifurcations in a predator-prey system of Leslie type with generalized Holling type III functional response. J. Differ. Equ. 257(6), 1721-1752 (2014)

5. Plesset, MS: The dynamics of cavitation bubbles. J. Appl. Mech. 16, 228-231 (1949)

6. Habets, P, Sanchez, L: Periodic solutions of some Liénard equations with singularities. Proc. Am. Math. Soc. 109, 1035-1044 (1990)

7. Tanaka, K: A note on generalized solutions of singular Hamiltonian systems. Proc. Am. Math. Soc. 122, 275-284 (1994)

8. Terracini, S: Remarks on periodic orbits of dynamical systems with repulsive singularities. J. Funct. Anal. 111, 213-238 (1993)

9. Gaeta, S, Manásevich, R: Existence of a pair of periodic solutions of an ode generalizing a problem in nonlinear elasticity via variational methods. J. Math. Anal. Appl. 123, 257-271 (1988)

10. Fonda, A: Periodic solutions for a conservative system of differential equations with a singularity of repulsive type. Nonlinear Anal. 24, 667-676 (1995)

11. Jebelean, P, Mawhin, J: Periodic solutions of singular nonlinear differential perturbations of the ordinary $p$-Laplacian. Adv. Nonlinear Stud. 2(3), 299-312 (2002)

12. Torres, PJ: Mathematical Models with Singularities - A Zoo of Singular Creatures. Atlantis Press, Amsterdam (2015). ISBN:978-94-6239-105-5

13. Nagumo, M: On the periodic solution of an ordinary differential equation of second order. In: Zenkoku Shijou Suugaku Danwakai, pp. $54-61$ (1944) (in Japanese). English translation in Mitio Nagumo Collected Papers, Sringer, Berlin (1993)

14. Derwidué, L: Systemes différentiels non linéaires ayant solutions périodiques. Acad. R. Belg., Cl. Lett. Sci., V Ser. 49, 11-32 (1963)

15. Fauré, R: Solutions périodiques d'équations différentielles et méthode de Leray-Schauder. Ann. Inst. Fourier 14(1), 195-204 (1964)

16. Gordon, WB: Conservative dynamical systems involving strong forces. Trans. Am. Math. Soc. 204, 113-135 (1975)

17. Lazer, AC, Solimini, S: On periodic solutions of nonlinear differential equations with singularities. Proc. Am. Math. Soc. 99, 109-114 (1987)

18. Torres, PJ: Existence of one-signed periodic solutions of some second order differential equations via a Krasnoselskii fixed point theorem. J. Differ. Equ. 190, 643-662 (2003)

19. Torres, PJ, Zhang, M: Twist periodic solutions of repulsive singular equations. Nonlinear Anal. 56, 591-599 (2004)

20. Torres, PJ: Bounded solutions in singular equations of repulsive type. Nonlinear Anal. 32, 117-125 (1998)

21. Jiang, $\mathrm{D}, \mathrm{Chu}, \mathrm{J}$, Zhang, M: Multiplicity of positive periodic solutions to superlinear repulsive singular equations. J. Differ. Equ. 211, 282-302 (2005)

22. del Pino, M, Manásevich, R: Infinitely many T-periodic solutions for a problem arising in nonlinear elasticity. J. Differ. Equ. 103, 260-277 (1993)

23. del Pino, M, Manásevich, R, Montero, A: T-Periodic solutions for some second order differential equations with singularities. Proc. R. Soc. Edinb., Sect. A 120(3-4), 231-243 (1992)

24. Fonda, A, Manasevich, R, Zanolin, F: Subharmonics solutions for some second order differential equations with singularities. SIAM J. Math. Anal. 24, 1294-1311 (1993)

25. Torres, PJ: Weak singularities may help periodic solutions to exist. J. Differ. Equ. 232, 277-284 (2007)

26. Chu, J, Torres, PJ, Zhang, M: Periodic solutions of second order non-autonomous singular dynamical systems. J. Differ. Equ. 239, 196-212 (2007)

27. Li, X, Zhang, Z: Periodic solutions for second order differential equations with a singular nonlinearity. Nonlinear Anal. 69, 3866-3876 (2008) 
28. Hakl, R, Torres, PJ, Zamora, M: Periodic solutions of singular second order differential equations: upper and lower functions. Nonlinear Anal. 74, 7078-7093 (2011)

29. Hakl, R, Torres, PJ: On periodic solutions of second order differential equations with attractive-repulsive singularities J. Differ. Equ. 248, 111-126 (2010)

30. Zhang, M: Periodic solutions of Liénard equations with singular forces of repulsive type. J. Math. Anal. Appl. 203(1), 254-269 (1996)

31. Martins, R: Existence of periodic solutions for second-order differential equations with singularities and the strong force condition. J. Math. Anal. Appl. 317, 1-13 (2006)

32. Wang, Z: Periodic solutions of Liénard equation with a singularity and a deviating argument. Nonlinear Anal., Real World Appl. 16(1), 227-234 (2014)

33. Hakl, R, Torres, PJ, Zamora, M: Periodic solutions of singular second order differential equations: the repulsive case. Topol. Methods Nonlinear Anal. 39, 199-220 (2012)

34. Lu, S: A new result on the existence of periodic solutions for Liénard equations with a singularity of repulsive type. J. Inequal. Appl. 2017, 37 (2017). doi:10.1186/s13660-016-1285-8

35. Lu, S, Zhong, T, Gao, Y: Periodic solutions of $p$-Laplacian equations with singularities. Adv. Differ. Equ. 2016, 146 (2016). doi:10.1186/s13662-016-0875-6

36. Manásevich, R, Mawhin, J: Periodic solutions for nonlinear systems with $p$-Laplacian-like operators. J. Differ. Equ. 145 367-393 (1998)

\section{Submit your manuscript to a SpringerOpen ${ }^{\circ}$ journal and benefit from:}

- Convenient online submission

- Rigorous peer review

- Open access: articles freely available online

- High visibility within the field

- Retaining the copyright to your article

Submit your next manuscript at $\boldsymbol{\nabla}$ springeropen.com 